\title{
Consensus Based Platoon Algorithm for Velocity-Measurement-Absent Vehicles with Actuator Saturation
}

\author{
Maode Yan, Ye Tang, Panpan Yang, and Lei Zuo \\ School of Electronic and Control Engineering, Chang'an University, Xian 710064, China \\ Correspondence should be addressed to Maode Yan; mdyan@chd.edu.cn
}

Received 1 June 2017; Accepted 19 July 2017; Published 20 August 2017

Academic Editor: Cheng S. Chin

Copyright (C) 2017 Maode Yan et al. This is an open access article distributed under the Creative Commons Attribution License, which permits unrestricted use, distribution, and reproduction in any medium, provided the original work is properly cited.

\begin{abstract}
We investigate the vehicle platoon problems, where the actuator saturation and absent velocity measurement are taken into consideration. Firstly, a novel algorithm, where a smooth function is introduced to deal with the sharp corner of the input signals, is proposed for a group of vehicles with actuator saturation by using the consensus theory. Secondly, by applying an auxiliary system for the followers to estimate the velocities, a control strategy for the vehicle platoon with actuator saturation and absent velocity measurement is designed via the adaptive control approach. Finally, numerical simulations are provided to illustrate the effectiveness of the proposed approaches.
\end{abstract}

\section{Introduction}

Vehicle platoon is well studied in the field of formation control, which is an effective approach to enhance the traffic safety and efficiency [1]. The main objective of vehicle platoon is to avoid traffic jams and improve the traffic security. Usually, a vehicle platoon contains a group of vehicles, which are moving in a lane with certain velocities. From this definition, we can clearly find that the key point of vehicle platoon control is to adjust the velocities and positions of the vehicles, such that they can reach the desired platoon.

In the past few years, a lot of results, like sliding mode control [2], consensus [3], neural network [4], and so on [5-7], have been proposed to deal with the platoon control problems in some ways. For instance, a robust acceleration tracking control with vehicle longitudinal dynamics is presented in [8] for platoon-level automation. In [9], an adaptive bidirectional platoon control method is proposed by using a coupled sliding mode control algorithm. Furthermore, a novel distributed control architecture is given in [10] for heterogeneous platoons, where a group of linear timeinvariant autonomous vehicles are considered.

To further study the vehicle platoon control problems, some limitations are taken into account. For example, in [11], range-limit sensors and delayed actuators are handled by guaranteed-cost platoon control method. Considering the capacity limitation and random packet loss, a closed-form methodology for vehicular platoon control is proposed in [12]. Moreover, a hierarchical platoon control framework is established in [13] to deal with the negative effect of the tracking parameter. The practical string stability of platoon is investigated in [14] for parasitic time delays and lags of the actuators and sensors. In [15], the authors propose control laws in the case of losing communications between the lead vehicle and the other vehicles in a platoon. Besides, disturbances, nonlinear, control input nonlinearities are also taken into consideration in many literatures [16, 17]. However, the fundamental limitations, associated with both actuator saturation and absent velocity measurement, have not been fully studied.

Actuator saturation is one of the most common constraints in practical control systems and it is still a challenge to design the control law with actuator saturation. Considerable attention is paid to the systems with actuator saturation since 1950s [18]. In [19], an online approximation is developed for automation train operation with the presence of actuator saturation. In [20], a position and heading trajectory tracking control law is developed for Swedish wheeled robots with 


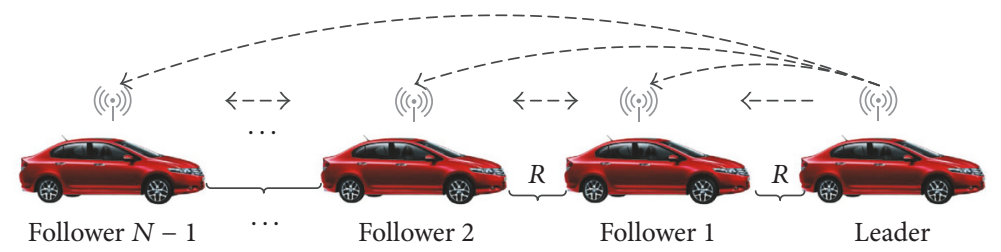

FIGURE 1: Topology structure of vehicle platoon.

unaccounted actuator saturation. In [21], a modified fault tolerant control strategy is presented in the flexible spacecraft to ensure that the control signal will never incur saturation.

For the vehicle platoon with absent velocity measurement, there are also some results. For instance, in [22], passive filters are employed to design an output feedback coordinated formation controller in multiple spacecrafts with absent velocity measurement. A decentralized control strategy is proposed with the neighboring topology for velocity constraints in double-integrator dynamics in [23]. In [24], an adaptive MIMO-ESO is designed to estimate the unmeasurable linear and angular velocities of underwater robots. Moreover, leader-follower output feedback synchronization scheme is given in [25] for controlling the attitude of two satellites when angular velocity measurements are not available. However, to the best of our knowledge, few results have dealt with both issues, absent velocity measurement and actuator saturation, simultaneously in vehicle platoon control.

Motivated by this fact, we propose a novel control strategy to dispose of both the cases: actuator saturation and absent velocity measurement in vehicle platoon. The main contributions of this paper are twofolds:

(i) We solve the "actuator saturation" problem in vehicle platoon, which occurs when the desired force exceeds the maximum output force of the engines. Transient period or even long period of such behavior will increase the loss of engine and reduce its life. A smooth function $\tanh (\cdot)$ is introduced to handle this problem such that the control inputs can always be below the maximum inputs.

(ii) We solve the "velocity absent measurement" problem in vehicle platoon control, where intensive oscillation may arise without velocity measurement. We apply an auxiliary system into the control law to estimate the velocity values so that the vehicles can relax the information requirements and communication burden.

The remainder of the paper is organized as follows. Section 2 gives the problem formulation. In Section 3, the controller for vehicle platoon control systems with actuator saturation is designed. Section 4 further proposes an algorithm with both actuator saturation and absent velocity measurement. Numerical simulations are presented in Section 5. Finally, Section 6 draws the conclusion.

\section{Problem Formulation}

Modeling is an important step in understanding the behavior of the system [26]. In this paper, we consider a group of vehicles, which are composed of one leader and $N-1$ followers, moving in one-dimensional plane. The topological structure of this vehicle platoon is shown in Figure 1, where $R$ is the desired distance between two consecutive vehicles.

The dynamics of the leader is shown as

$$
\dot{r}_{L}=v_{L}
$$

where $r_{L}$ and $v_{L}$ are the position and velocity of the leader, respectively.

The dynamic models of followers are described as

$$
\begin{aligned}
& \dot{r}_{i}=v_{i}, \\
& \dot{v}_{i}=\frac{1}{M_{i}} u_{i}, \\
& \quad i=1,2, \ldots, N-1,
\end{aligned}
$$

where $r_{i} \in \mathbb{R}^{m}$ and $v_{i} \in \mathbb{R}^{m}$ are the position and velocity of vehicle $i$, respectively. $M_{i}$ represents the mass of vehicle $i$ and $u_{i} \in \mathbb{R}^{m}$ denotes the control input.

In practical applications, the engine will work at maximum output status when there is actuator saturation, which will increase the loss of engine and reduce its life. In addition, due to the loss of velocity information, namely, "absent velocity measurement," the performance of vehicle platoon control will degrade.

Therefore, the main objective of this paper is to control the velocity-measurement-absent vehicles with actuator saturation such that they can move along a platoon with the desired velocities. Meanwhile, they can keep distance from avoiding collision during the process and the safe distances can converge to a stable constant when they reach the desired states.

\section{Vehicle Platoon with Actuator Saturation}

Due to the physical limitations of actuators, the control inputs $u_{i}$ can be written as

$$
\begin{aligned}
u_{i}(t) & =\operatorname{sat}\left(-u_{M}, u(t), u_{M}\right) \\
& = \begin{cases}\operatorname{sign}(u(t)) u_{M}, & |u(t)| \geq u_{M} \\
u(t), & |u(t)|<u_{M},\end{cases}
\end{aligned}
$$

where $u_{M}$ is the upper bound of $u_{i}(t)$. 
As there is a sharp corner between $u_{i}(t)$ and $u(t)$, it is unrealizable to use this kind of input signals in practice. Hence, we use a smooth function to approximate the control inputs, which is defined as [27]

$$
\begin{aligned}
g(u(t)) & =u_{M} \times \tanh \left(\frac{u(t)}{u_{M}}\right) \\
& =u_{M} \frac{e^{u(t) / u_{M}}-e^{-u(t) / u_{M}}}{e^{u(t) / u_{M}}+e^{-u(t) / u_{M}}} .
\end{aligned}
$$

Then, sat $(u(t))$ in (3) is expressed as

$$
\begin{aligned}
\operatorname{sat}(u(t)) & =g(u(t))+d_{1}(u(t)) \\
& =u_{M} \times \tanh \left(\frac{u(t)}{u_{M}}\right)+d_{1}(u(t)),
\end{aligned}
$$

where $d_{1}(u(t))=\operatorname{sat}(u(t))-g(u(t))$ is a bounded function and its bound can be obtained as

$$
\begin{aligned}
\left|d_{1}(u(t))\right| & =|\operatorname{sat}(u(t))-g(u(t))| \\
& \leq u_{M}(1-\tanh (1))=D_{1} .
\end{aligned}
$$

As $0 \leq|u(t)| \leq u_{M}$, the bound $d_{1}(t)$ increases from 0 to $D_{1}$ as $|u(t)|$ varies from 0 to $u_{M}$. Otherwise, the bound $d_{1}(u(t))$ decreases from $D_{1}$ to 0 .

On this basis, an algorithm for the vehicle platoon with actuator saturation is proposed as follows:

$$
\begin{aligned}
u_{i}= & \dot{v}_{r} \\
& -\underbrace{\sum_{j=1}^{n} k_{i j} \tanh \left(\lambda^{k} R_{i j}\right)}_{(\mathrm{a})} \\
& -\underbrace{\sum_{j=1}^{n} \gamma_{i j} \tanh \left[\lambda^{\gamma}\left(v_{i}-v_{j}\right)\right] .}_{(\mathrm{b})}
\end{aligned}
$$

Section (a) represents the position control, where $R_{i j}=$ $\left(r_{i}-r_{j}\right)-\left(\delta_{i}-\delta_{j}\right)$ and $\left(\delta_{i}-\delta_{j}\right)$ represents the desired distance between each two vehicles. In addition, $K=\left[k_{i j}\right]_{n \times n}$ is the weighted adjacency matrix with $k_{i j} \geq 0$ and $\lambda^{k}$ is the strictly positive scalar gain.

Section (b) is the velocity control, where $\gamma=\left[\gamma_{i j}\right]_{n \times n}$ is also the weighted adjacency matrix with $\gamma_{i j} \geq 0$ and $\lambda^{\gamma}$ is the strictly positive scalar gain.

Furthermore, the following lemma is introduced for the proposed control system.

Lemma 1. Suppose $\eta \in \mathbb{R}^{m}, \xi \in \mathbb{R}^{m}, \rho \in \mathbb{R}^{m}$, and $K=\left[k_{i j}\right] \in$ $\mathbb{R}^{n \times n}$. If $K$ is symmetric $\left(k_{i j}=k_{j i}\right)$, then

$$
\begin{gathered}
\frac{1}{2} \sum_{i=1}^{n} \sum_{j=1}^{n} k_{i j}\left(\eta_{i}-\eta_{j}\right)^{T} \tanh \left[\rho\left(\xi_{i}-\xi_{j}\right)\right] \\
=\sum_{i=1}^{n} \sum_{j=1}^{n} k_{i j} \eta_{i}^{T} \tanh \left[\rho\left(\xi_{i}-\xi_{j}\right)\right] .
\end{gathered}
$$

Then, the theorem of platoon control with actuator saturation is presented as follows.

Theorem 2. Consider a group of vehicles with actuator saturation in a one-dimensional platoon. By using the control law in (7), the vehicles can reach the platoon with desired distances and velocities. Namely,

$$
\begin{aligned}
& \text { (i) }\left|u_{i}\right| \leq\left|\dot{v}_{r}\right|_{\text {max }}+\left|\sum_{j=1}^{n} k_{i j}\right|+\left|\sum_{j=1}^{n} \gamma_{i j}\right| \text {; } \\
& \text { (ii) } \lim _{t \rightarrow \infty}\left(v_{i}-v_{j}\right)=\lim _{t \rightarrow \infty}\left(v_{i}-v_{L}\right)=0 \text {; } \\
& \text { (iii) } \lim _{t \rightarrow \infty}\left(r_{i}-r_{j}\right)=\|i-j\| R . \\
& \text { (iv) } \lim _{t \rightarrow \infty}\left(r_{i}-r_{L}\right)=i \cdot R .
\end{aligned}
$$

Proof. Based on absolute value inequality, we can easily find that the control input in (7) is bounded as

$$
\left|u_{i}\right| \leq\left|\dot{v}_{r}\right|_{\max }+\left|\sum_{j=1}^{n} k_{i j}\right|+\left|\sum_{j=1}^{n} \gamma_{i j}\right| .
$$

Let $\widetilde{v}_{i}=v_{i}-v_{r}$. Consider the following Lyapunov function candidate:

$$
V_{1}=\frac{1}{2} \sum_{i=1}^{n} \widetilde{v}_{i}^{\mathrm{T}} \widetilde{v}_{i}+\frac{1}{2} \sum_{i=1}^{n} \sum_{j=1}^{n} \frac{k_{i j}}{\lambda^{k}} \mathbf{1}_{m}^{\mathrm{T}} \log \left(\cosh \left(\lambda^{k} R_{i j}\right)\right),
$$

where $\mathbf{1}_{m} \in \mathbb{R}^{m}$ is a vector with all elements equal to one.

Note that $V_{1}$ is positive definite and radially unbound with respect to $R_{i j}$. Taking the derivative of $V_{1}$, we have

$$
\begin{aligned}
\dot{V}_{1} & =\sum_{i=1}^{n} \widetilde{v}_{i}^{\mathrm{T}} \dot{\widetilde{v}}_{i}+\frac{1}{2} \sum_{i=1}^{n} \sum_{j=1}^{n} k_{i j}\left(v_{i}-v_{j}\right)^{\mathrm{T}} \tanh \left(\lambda^{k} R_{i j}\right) \\
& =\sum_{i=1}^{n} \widetilde{v}_{i}^{\mathrm{T}}\left[-\sum_{j=1}^{n} k_{i j} \tanh \left(\lambda^{k} R_{i j}\right)\right. \\
& -\sum_{j=1}^{n} \gamma_{i j} \tanh \left[\lambda^{\gamma}\left(v_{i}-v_{j}\right)\right]+\frac{1}{2} \sum_{i=1}^{n} \sum_{j=1}^{n} k_{i j}\left(v_{i}-v_{j}\right)^{\mathrm{T}} \\
& \cdot \tanh \left(\lambda^{k} R_{i j}\right) .
\end{aligned}
$$

According to Lemma 1, we obtain that

$$
\dot{V}_{1}=-\frac{1}{2} \sum_{i=1}^{n} \sum_{j=1}^{n} \gamma_{i j}\left(\widetilde{v}_{i}-\widetilde{v}_{i}\right)^{\mathrm{T}} \tanh \left[\lambda^{\gamma}\left(\widetilde{v}_{i}-\widetilde{v}_{j}\right)\right] \leq 0 .
$$

This reveals that $V_{1} \leq V_{1}(0)$, and hence $v_{i}, r_{i}-r_{j}$ are globally bounded.

Since $\left(\dot{\widetilde{v}}_{i}-\dot{\tilde{v}}_{j}\right)=\left(\dot{v}_{i}-\dot{v}_{j}\right)$ is bounded, we can conclude that $\ddot{V}_{1}$ is bounded. Based on Barbălat Lemma, we have $\lim _{t \rightarrow \infty}\left(\widetilde{v}_{i}-\widetilde{v}_{j}\right)=0$ and $\lim _{t \rightarrow \infty}\left(v_{i}-v_{j}\right)=0$.

As $\lim _{t \rightarrow \infty}\left(\dot{v}_{i}-\dot{v}_{r}\right)=\lim _{t \rightarrow \infty}\left(u_{i}-\dot{v}_{r}\right)=0$, it leads to

$$
\begin{gathered}
\lim _{t \rightarrow \infty}\left[-\sum_{j=1}^{n} \gamma_{i j} \tanh \left[\lambda^{\gamma}\left(v_{i}-v_{j}\right)\right]\right. \\
\left.-\sum_{j=1}^{n} k_{i j} \tanh \left(\lambda^{k} R_{i j}\right)\right]=0 .
\end{gathered}
$$


Then, we have

$$
\lim _{t \rightarrow \infty} \sum_{j=1}^{n} k_{i j} \tanh \left(\lambda^{k} R_{i j}\right)=0 .
$$

Eq. (14) satisfies

$$
\begin{aligned}
& \lim _{t \rightarrow \infty} \sum_{i=1}^{n} \sum_{j=1}^{n} k_{i j}\left(r_{i}-\delta_{i}\right)^{\mathrm{T}} \tanh \left(\lambda^{k} R_{i j}\right) \\
& =\frac{1}{2} \lim _{t \rightarrow \infty} \sum_{i=1}^{n} \sum_{j=1}^{n} k_{i j} R_{i j}^{\mathrm{T}} \tanh \left(\lambda^{k} R_{i j}\right)=0 .
\end{aligned}
$$

As a result, $\lim _{t \rightarrow \infty} R_{i j}=0$.

Remark 3. The purpose of using $\left(\delta_{i}-\delta_{j}\right)$ as a safe distance is to complete the proof by Lyapunov function. With the algorithm (7) proposed in this section, we can obtain that $\left(r_{i}-r_{j}\right) \rightarrow$ $\left(\delta_{i}-\delta_{j}\right) \rightarrow\|i-j\| R$.

\section{Vehicle Platoon with Actuator Saturation and Absent Velocity Measurement}

In this section, we take both the actuator saturation and absent velocity measurement into consideration and propose a novel platoon control law. The main strategy of this proposed method is to design an auxiliary system for the followers, so that they can estimate the velocities effectively.

Invoking the adaptive control theory, the algorithm for the vehicle platoon with both actuator saturation and absent velocity measurement can be presented as

$$
\begin{aligned}
u_{i}= & \dot{v}_{r}-\sum_{j=1}^{n} k_{i j} \tanh \left(\lambda^{k} R_{i j}\right)-\zeta_{i}^{v} \tanh \left(\lambda^{\zeta}\left(r_{i}-\Psi_{i}\right)\right) \\
& +\dot{\Theta}_{i},
\end{aligned}
$$

where $K=\left[k_{i j}\right]_{n \times n}$ is the weighted adjacency matrix with $k_{i j} \geq 0 . \lambda^{k}$ and $\lambda^{\zeta}$ are positive scalar gains, and $\zeta_{i}^{v}>0$ is defined as the scalar gain. follows:

The auxiliary system, denoted by $\Theta_{i}$ and $\Psi_{i}$, is defined as

$$
\begin{aligned}
\dot{\Theta}_{i}= & -\sum_{j=1}^{n} k_{i j} \tanh \left(\lambda^{k} R_{i j}\right)-k_{i}^{\theta} \tanh \left(\lambda^{\theta} \Theta_{i}\right) \\
& -\zeta_{i}^{v} \tanh \left(\lambda^{\zeta}\left(r_{i}-\Psi_{i}\right)\right), \\
\dot{\Psi}_{i}= & v_{r}+k_{i}^{\psi}\left(r_{i}-\Psi_{i}\right),
\end{aligned}
$$

where $\lambda^{\theta}, k_{i}^{\theta}$, and $k_{i}^{\psi}$ are strictly positive constants, and $\Theta_{i}$ and $\Psi_{i}$ have arbitrarily initial values.

Theorem 4. Consider a group of vehicles with actuator saturation and absent velocity measurement in a platoon. By using the control law in (16), the vehicles can reach the platoon with desired distances and velocities. Namely, (i) $\left|u_{i}\right| \leq\left|\dot{v}_{r}\right|_{\max }+2 \times\left[\sum_{j=1}^{n}\left(\left|k_{i j}\right|+\left|\zeta_{i}^{v}\right|\right)\right]+\left|k_{i}^{\theta}\right|$;

(ii) $\lim _{t \rightarrow \infty}\left(v_{i}-v_{j}\right)=\lim _{t \rightarrow \infty}\left(v_{i}-v_{L}\right)=0$;

(iii) $\lim _{t \rightarrow \infty}\left(r_{i}-r_{j}\right)=\|i-j\| R$.

(iv) $\lim _{t \rightarrow \infty}\left(r_{i}-r_{L}\right)=i \cdot R$.

Proof. From Theorem 2, we can obtain that the control laws in (16) are bounded as

$$
\left|u_{i}\right| \leq\left|\dot{v}_{r}\right|_{\max }+2 \times\left[\sum_{j=1}^{n}\left(\left|k_{i j}\right|+\left|\zeta_{i}^{v}\right|\right)\right]+\left|k_{i}^{\theta}\right| .
$$

Then denote $\tilde{v}_{i}=v_{i}-v_{r}$. Consider the following Lyapunov function candidate

$$
\begin{aligned}
V_{2}= & \frac{1}{2} \sum_{i=1}^{n}\left(\widetilde{v}_{i}-\Theta_{i}\right)^{\mathrm{T}}\left(\widetilde{v}_{i}-\Theta_{i}\right)+\frac{1}{2} \sum_{i=1}^{n} \Theta_{i}^{\mathrm{T}} \Theta_{i} \\
& +\sum_{i=1}^{n} \frac{\zeta_{i}^{v}}{\lambda^{\zeta}} \mathbf{1}_{m}^{\mathrm{T}} \log \left(\cosh \left(\lambda^{\zeta}\left(r_{i}-\Psi_{i}\right)\right)\right) \\
& +\frac{1}{2} \sum_{i=1}^{n} \sum_{j=1}^{n} \frac{k_{i j}}{\lambda^{k}} \mathbf{1}_{m}^{\mathrm{T}} \log \left[\cosh \left(\lambda^{k} R_{i j}\right)\right],
\end{aligned}
$$

where $\mathbf{1}_{m} \in \mathbb{R}^{m}$ is a vector with all elements equal to one and the functions $\log (\cdot)$ and $\cosh (\cdot)$ are defined elements-wise for a vector.

Invoking (2) and (16), the time derivative of $V_{2}$ is given by

$$
\begin{aligned}
\dot{V}_{2} & =\sum_{i=1}^{n}\left(\widetilde{v}_{i}-\Theta_{i}\right)^{\mathrm{T}}\left(\dot{\vec{v}}_{i}-\dot{\Theta}_{i}\right)+\sum_{i=1}^{n} \Theta_{i}^{\mathrm{T}} \dot{\Theta}_{i}+\sum_{i=1}^{n} \zeta_{i}^{v}\left(\dot{r}_{i}\right. \\
& \left.-\dot{\Psi}_{i}\right)^{\mathrm{T}} \tanh \left(\lambda^{\zeta}\left(r_{i}-\Psi_{i}\right)\right)+\frac{1}{2} \sum_{i=1}^{n} \sum_{j=1}^{n} k_{i j}\left(\dot{R}_{i j}\right)^{\mathrm{T}} \\
& \cdot \tanh \left(\lambda^{k} R_{i j}\right)=\sum_{i=1}^{n}\left(\widetilde{v}_{i}-\Theta_{i}\right)^{\mathrm{T}} \\
& \cdot\left[\sum_{j=1}^{n} k_{i j} \tanh \left(\lambda^{k} R_{i j}\right)-\zeta_{i}^{v} \tanh \left(\lambda^{\zeta}\left(r_{i}-\Psi_{i}\right)\right)\right] \\
& +\sum_{i=1}^{n} \Theta_{i}^{\mathrm{T}}\left[-k_{i}^{\theta} \tanh \left(\lambda^{\theta} \Theta_{i}\right)-\sum_{j=1}^{n} k_{i j} \tanh \left(\lambda^{k} R_{i j}\right)\right. \\
& \left.-\zeta_{i}^{v} \tanh \left(\lambda^{\zeta}\left(r_{i}-\Psi_{i}\right)\right)\right]-\sum_{i=1}^{n} \zeta_{i}^{v} k_{i}^{\psi}\left(r_{i}-\Psi_{i}\right)^{\mathrm{T}} \\
& +\frac{1}{2} \sum_{i=1}^{n} \sum_{j=1}^{n} k_{i j}\left(v_{i}-v_{j}\right)^{\mathrm{T}} \tanh \left(\lambda^{k} R_{i j}\right) . \\
& \tanh \left(\lambda^{\zeta}\left(r_{i}-\Psi_{i}\right)\right)+\sum_{i=1}^{n} \zeta_{i}^{v} \widetilde{v}_{i} \tanh \left(\lambda^{\zeta}\left(r_{i}-\Psi_{i}\right)\right)
\end{aligned}
$$


From Lemma 1, we know that $\widetilde{v}_{i}=v_{i}-v_{r}$ and $v_{i}-v_{j}=$ $\widetilde{v}_{i}-\widetilde{v}_{j}$. Then, we obtain that

$$
\begin{aligned}
\dot{V}_{2}= & -\sum_{i=1}^{n} \zeta_{i}^{v} k_{i}^{\psi}\left(r_{i}-\Psi_{i}\right)^{\mathrm{T}} \tanh \left(\lambda^{\zeta}\left(r_{i}-\Psi_{i}\right)\right) \\
& -\sum_{i=1}^{n} k_{i}^{\theta} \Theta_{i}^{\mathrm{T}}\left[\tanh \left(\lambda^{\theta} \Theta_{i}\right)\right] \leq 0 .
\end{aligned}
$$

This shows that $V_{2} \leq V_{2}(0)$, and $v_{i}, \Theta_{i}, R_{i j}$, and $\left(r_{i}-\Psi_{i}\right)$ are globally bounded.

From the above derivations, we conclude that $\dot{\Psi}$ is bounded. In addition, due to the boundedness of $\tanh (\cdot), \dot{\Theta}_{i}$ and $\ddot{V}_{2}$ are also bounded. On the basis of the above analysis, we obtain that $\lim _{t \rightarrow \infty} \Theta_{i}(t)=0$ and $\lim _{t \rightarrow \infty}\left(r_{i}(t)-\Psi_{i}(t)\right)=$ 0 . Since $\dot{\Psi}_{i}, v_{i}$, and $\dot{\Theta}_{i}$ are bounded, we know that $\ddot{\Theta}_{i}$ is also bounded. From Barbălat Lemma, we can testify that $\lim _{t \rightarrow \infty} \dot{\Theta}_{i}(t)=0$ with the fact that $\lim _{t \rightarrow \infty} \Theta_{i}(t)=0$ and $\ddot{\Theta}_{i}$ is bounded.

Then, we have that

$$
\lim _{t \rightarrow \infty} \sum_{n=1}^{n} k_{i j} \tanh \left(\lambda^{k} R_{i j}\right)=0 .
$$

With $k_{i j}=k_{j i}$, (23) satisfies

$$
\begin{array}{r}
\lim _{t \rightarrow \infty} \sum_{i=1}^{n} k_{i j}\left(r_{i}-\delta_{i}\right)^{\mathrm{T}} \tanh \left(\lambda^{k} R_{i j}\right) \\
\quad=\frac{1}{2} \lim _{t \rightarrow \infty} \sum_{i=1}^{n} k_{i j} R_{i j}^{\mathrm{T}} \tanh \left(\lambda^{k} R_{i j}\right) .
\end{array}
$$

Therefore, $\lim _{t \rightarrow \infty} R_{i j}=0$. According to Barbălat Lemma, we can obtain that $\lim _{t \rightarrow \infty}\left(v_{i}-v_{j}\right)=0$ because $\dot{\tilde{v}}_{i}=\dot{v}_{i}-\dot{v}_{r}$ is bounded.

As the derivative of (18), $\ddot{\Psi}=\dot{v}_{r}+k_{i}^{\psi}\left(\dot{v}_{i}-\dot{\Psi}_{i}\right)$, is bounded and $u_{i}$ is also bounded, $\left(\ddot{r}_{i}-\ddot{\Psi}_{i}\right)$ is proven to be bounded. Then, we can conclude that $\lim _{t \rightarrow \infty}\left(r_{i}-\Psi_{i}\right)=0$, and $\lim _{t \rightarrow \infty}\left(v_{i}-\right.$ $\left.\dot{\Psi}_{i}\right)=0$. Furthermore, we know from (18) that $\lim _{t \rightarrow \infty}\left(v_{i}-\right.$ $\left.v_{r}\right)=0$.

Based on the above analysis, we can conclude that $\lim _{t \rightarrow \infty}\left(v_{i}-v_{r}\right)=0$ for all vehicles.

Remark 5. As the auxiliary system has the same structure with the actual velocity mode, the proposed approach in this paper provides an indirect asymptotic estimation of the velocity. By applying this auxiliary system, the platoon control system can generate the necessary damping for the overall closed stability [28, 29].

\section{Simulations}

To verify the effectiveness of the proposed algorithms in this paper for handing both the "actuator saturation" and "absent velocity measurement" problem, we design a series of simulations, which consist of 6 followers and 1 leader. The initial conditions and safe distance are shown in Table 1.
TABLE 1: Initial conditions and safe distance.

\begin{tabular}{lcc}
\hline Symbol & Value & Unit \\
\hline$\delta_{i}-\delta_{j}$ & 5 & $\mathrm{~m}$ \\
$r_{L}(0)$ & 38 & $\mathrm{~m}$ \\
$r_{i}(0)$ & {$[0 ; 10 ; 14 ; 20 ; 26 ; 31]$} & $\mathrm{m}$ \\
$v_{L}(0)$ & 0 & $\mathrm{~m} / \mathrm{s}$ \\
$v_{i}(0)$ & 0 & $\mathrm{~m} / \mathrm{s}$ \\
\hline
\end{tabular}

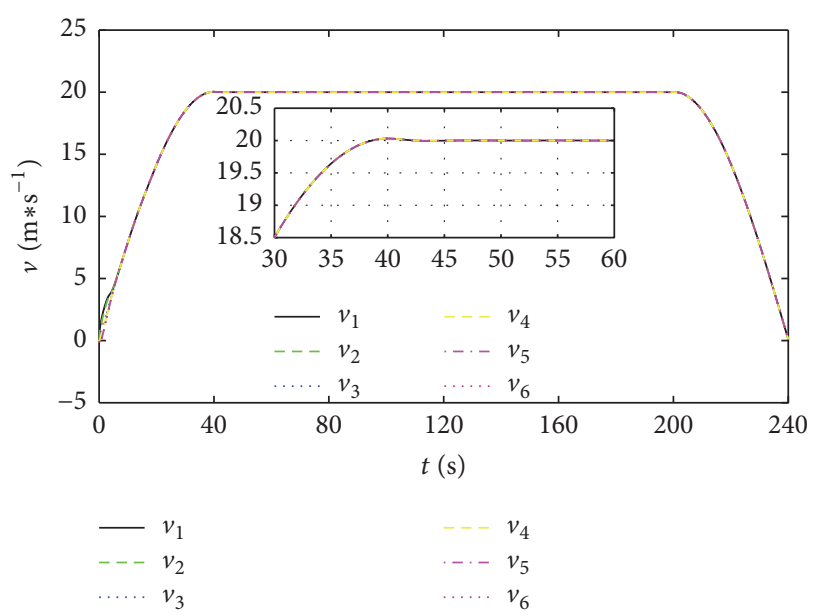

FIGURE 2: Velocities of vehicles with actuator saturation in vehicle platoon.

Then, the reference velocities of the leader are presented as follows, which includes the processes of acceleration, cruise, and deceleration:

$$
\begin{aligned}
& v_{r 1}=20 \sin \left(\frac{\pi}{80} t\right), \quad t<40 ; \\
& v_{r 2}=20, \quad 40 \leq t<200 ; \\
& v_{r 3}=20 \sin \left(\frac{\pi}{80} t\right), \quad 200 \leq t<240 .
\end{aligned}
$$

5.1. Vehicle Platoon with Actuator Saturation. In this part, we only consider the control strategy in (7) and select the controller gains such as $k_{i j}=\gamma_{i j}=\lambda^{k}=\lambda^{\gamma}=1$.

In the simulations, Figure 2 shows the velocities of vehicles, from which we can see that all the vehicles follow the desired velocities based on the present control law. In Figure 3, it is observed that the followers track the positions of leader vehicle and the distance between two consecutive vehicles converges to a desired constant value. In addition, we can see that there is no collision during this process.

Note that, in Figure 4, control laws generate traction force for vehicles during the acceleration phase, while they generate braking force when the desired velocities decline. In particularly, the maximum traction force and maximum braking force are limited. Aforementioned simulations confirm the effectiveness of our proposed approach with actuator saturation. 


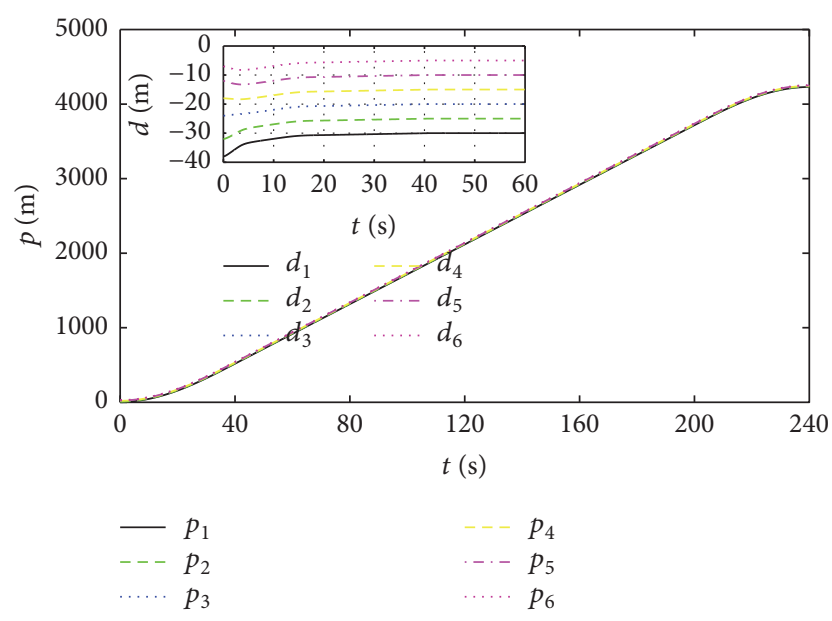

FIGURE 3: Positions of each vehicle and position gaps between vehicles.

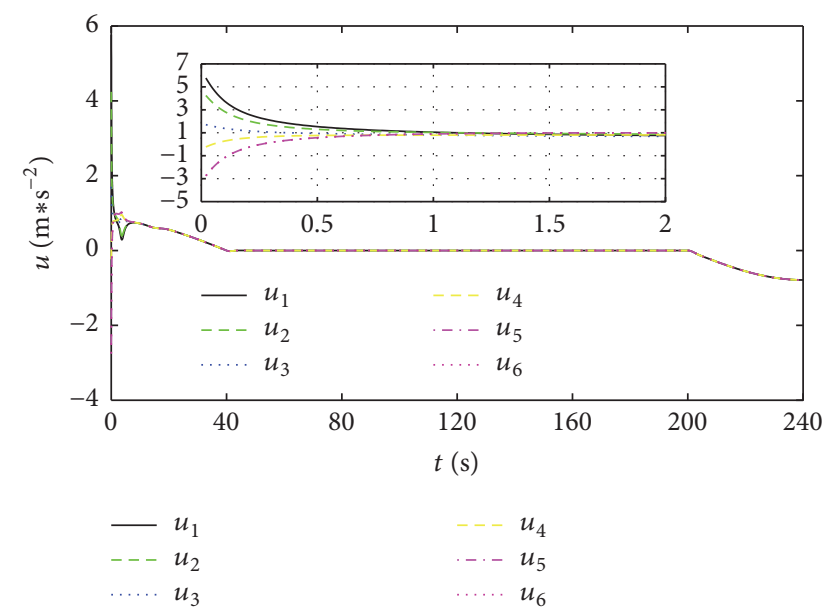

FIgURE 4: Control inputs of vehicles with actuator saturation in vehicle platoon.

5.2. Vehicle Platoon with Actuator Saturation and Absent Velocity Measurement. In this part, we consider control law (16)-(18). We set the initial values of signal $\Theta_{i}$ as $\Theta_{i}=0$ and $\dot{\Theta}_{i}$ as $\dot{\Theta}_{i}=0$. Similarly, $\Psi_{i}=\dot{\Psi}_{i}=0$. The controller gains are chosen as $\lambda^{k}=\lambda^{\zeta}=\zeta_{i}^{v}=1$. Figures 5-7 are depicted to validate the effectiveness of control algorithm in (16).

Velocities are illustrated in Figure 5, from which we can see that followers can reach the desired velocity, while oscillations present at the beginning in vehicle platoon. This is caused by the damping from auxiliary in order to handle the absent velocities. Figure 6 depicts the position trajectories of vehicles, which has the similar trend with Figure 3. As shown in Figure 6, position gaps converge to the desired value asymptotically, which indicates that platoon maneuver is achieved.

Control inputs in vehicle platoon with actuator saturation and absent velocity measurement are shown in Figure 7, where active control inputs exist in the first period of acceleration and then they can make a quick and smooth change

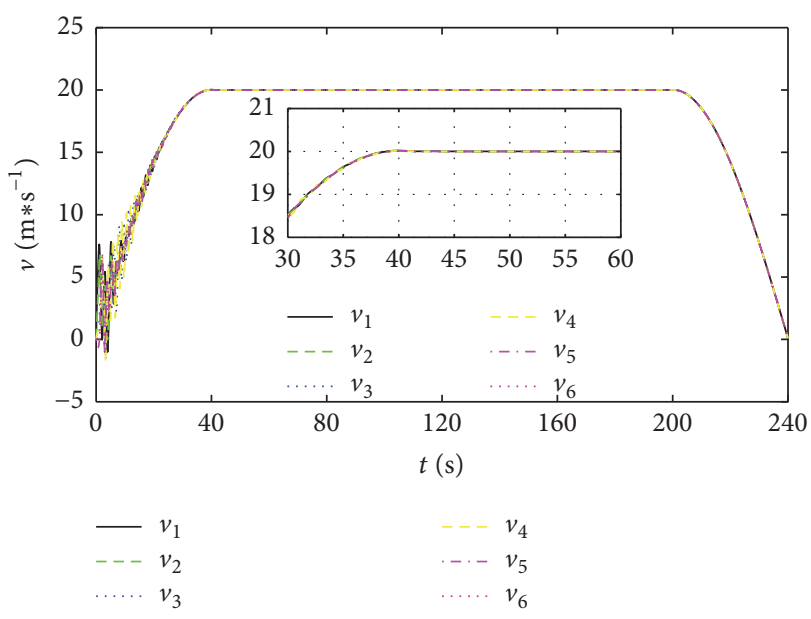

FIGURE 5: Velocities of vehicles with actuator saturation and absent velocity measurement.

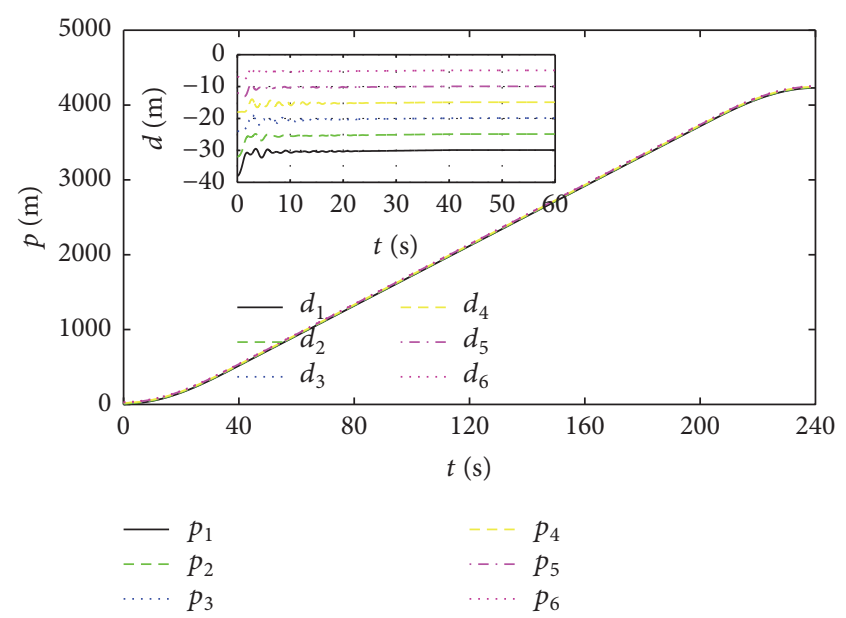

FIgURE 6: Positions of each vehicle and position gaps between vehicles.

to accommodate the various desired velocities. Furthermore, we can also see that maximum control input in Figure 7 is $12 \mathrm{~m} \cdot \mathrm{s}^{-2}$, which is within the controllable range in (19) due to the limitations of traction behavior and braking behavior. All the simulations in this part demonstrate control strategy in (16) can achieve similar control performance as control law in (7) despite absent velocity measurement.

5.3. Comparative Analysis. In order to illustrate the advantages of the proposed algorithm, we further design two comparative simulations between the proposed method in this paper and the other related methods.

Firstly, we verify the superiority of the algorithm with the smooth function $\tanh (\cdot)$ in Section 4. A comparison simulation is designed for the proposed algorithm in Section 4 with an existing algorithm, where the upper bounded input is $15 \mathrm{~m} \cdot \mathrm{s}^{-2}$, the lower bounded input is $-15 \mathrm{~m} \cdot \mathrm{s}^{-2}$, and other parameters are the same. Under the control of the algorithm 


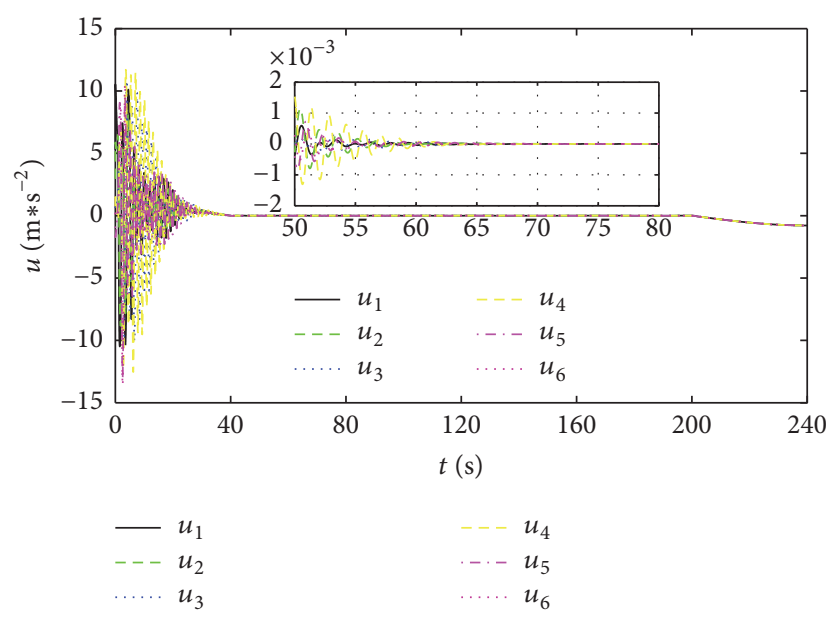

FIGURE 7: Control inputs of vehicles with actuator saturation and absent velocity measurement.

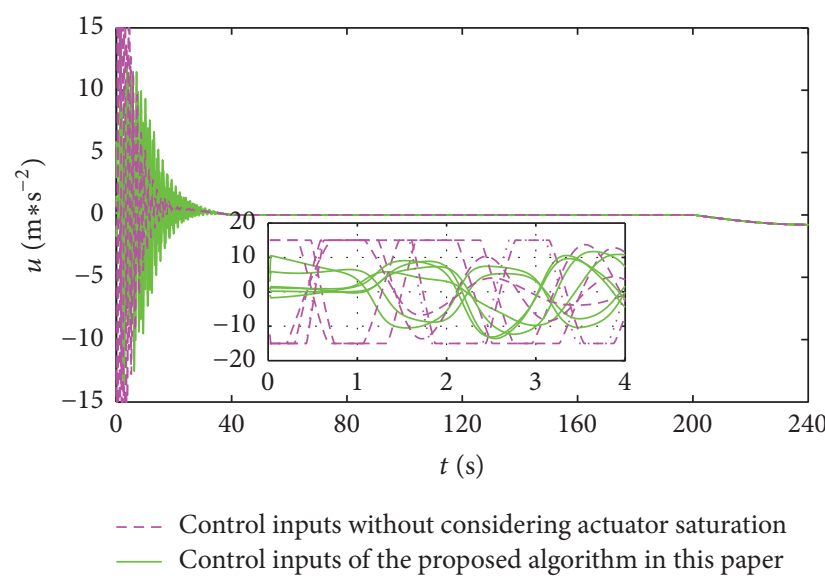

FIGURE 8: Performance comparison with actuator saturation.

in Section 4, the vehicle platoon can maintain the desired states without actuator saturation. The results are as follows.

From Figure 8, we can see that the proposed algorithm can deal with the control inputs saturation, while saturation occurs in other related algorithm.

Secondly, we verify the superiority of the algorithm with the auxiliary system in Section 4 for handling the absent velocity measurement problem. The velocity measurement can be estimated by the auxiliary system on the basis of position information. An existing algorithm without considering the absent velocity measurement is performed as the contrast. The results are as follows.

From Figure 9, we can see that, without velocity measurement, other algorithm will lead to intensive oscillation in the control inputs. However, the proposed algorithm in this paper can effectively deal with this problem and the control inputs can converge to zero within a short time. In addition, the velocities in some related literatures are varying during all the simulations.

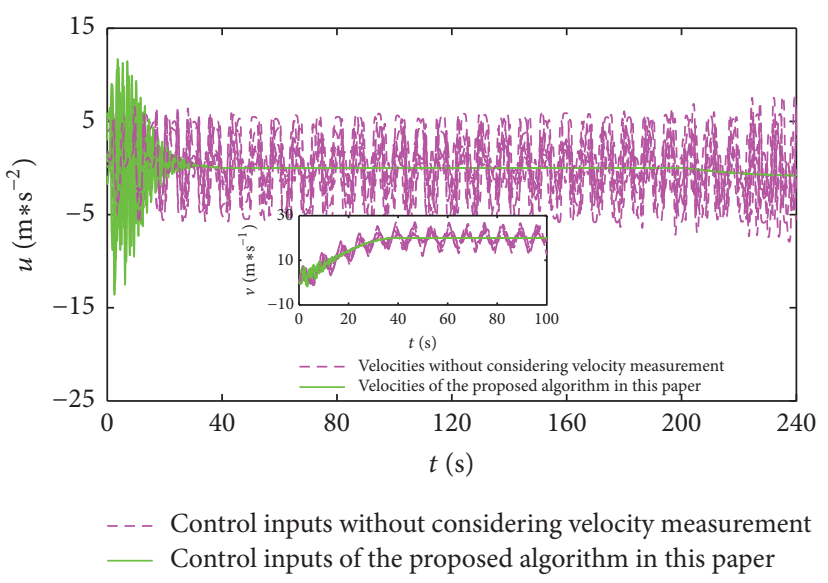

FIGURE 9: Performance comparison with absent velocity measurement.

\section{Conclusions}

This study proposed a method to investigate vehicle platoon with actuator saturation and absent velocity measurement. Firstly, we applied the consensus theory to the vehicle platoon control strategy with actuator saturation. Based on this achievement, we further proposed vehicle platoon control algorithm for the velocity-measurement-absent vehicles with actuator saturation. The stability of these proposed vehicle platoon systems was proved through the Lyapunov theorem. Finally, numerical simulations were provided to verify the proposed approaches.

\section{Conflicts of Interest}

The authors declare that there are no conflicts of interest regarding the publication of this paper.

\section{Acknowledgments}

This work is partially supported by the Fundamental Research Funds for the Central Universities of China (310832163403, 310832171004, and 310832101012), the Key Science and Technology Program of Shaanxi Province (2017JQ6060), and the China Postdoctoral Science Foundation Project (2017M613030).

\section{References}

[1] S. Solyom and E. Coelingh, "Performance limitations in vehicle platoon control," IEEE Intelligent Transportation Systems Magazine, vol. 5, no. 4, pp. 112-120, 2013.

[2] C. S. Chin and C. Wheeler, "Sliding-mode control of an electromagnetic actuated conveyance system using contactless sensing," IEEE Transactions on Industrial Electronics, vol. 60, no. 11, pp. 5315-5324, 2013.

[3] C. Santos, F. Espinosa, E. Santiso, M. Martinez, and M. Mazo, "Aperiodic consensus control for tracking nonlinear trajectories of a platoon of vehicles," in Proceedings of the 18th IEEE 
International Conference on Intelligent Transportation Systems (ITSC '15), pp. 1983-1988, IEEE, September 2015.

[4] R. Cui, B. Ren, and S. S. Ge, "Synchronised tracking control of multi-agent system with high-order dynamics," IET Control Theory \& Applications, vol. 6, no. 5, pp. 603-614, 2012.

[5] W. Lin and C. S. Chin, "Block diagonal dominant remotely operated vehicle model simulation using decentralized model predictive control," Advances in Mechanical Engineering, vol. 9, no. 4, pp. 1-24, 2017.

[6] Y.-F. Peng, C.-F. Hsu, C.-M. Lin, and T.-T. Lee, "Robust intelligent backstepping longitudinal control of vehicle platoons with Ho tracking performance," in Proceedings of the 2006 IEEE International Conference on Systems, Man and Cybernetics, pp. 4648-4653, IEEE, October 2006.

[7] C. S. Chin, M. W. S. Lau, E. Low, and G. G. L. Seet, "Robust and decoupled cascaded control system of underwater robotic vehicle for stabilization and pipeline tracking," Proceedings of the Institution of Mechanical Engineers. Part I: Journal of Systems and Control Engineering, vol. 222, no. 4, pp. 261-278, 2008.

[8] S. E. Li, F. Gao, D. Cao, and K. Li, "Multiple-Model Switching Control of Vehicle Longitudinal Dynamics for Platoon-Level Automation," IEEE Transactions on Vehicular Technology, vol. 65, no. 6, pp. 4480-4492, 2016.

[9] J.-W. Kwon and D. Chwa, "Adaptive bidirectional platoon control using a coupled sliding mode control method," IEEE Transactions on Intelligent Transportation Systems, vol. 15, no. 5, pp. 2040-2048, 2014.

[10] Ş. SabǍu, C. OarǍ, S. Warnick, and A. Jadbabaie, "Optimal distributed control for platooning via sparse coprime factorizations," in Proceedings of the 2016 American Control Conference (ACC '16), pp. 2591-2598, July 2016.

[11] G. Guo and W. Yue, "Autonomous platoon control allowing range-limited sensors," IEEE Transactions on Vehicular Technology, vol. 61, no. 7, pp. 2901-2912, 2012.

[12] G. Guo and S. Wen, "Communication scheduling and control of a platoon of vehicles in VANETs," IEEE Transactions on Intelligent Transportation Systems, vol. 17, no. 6, pp. 1551-1563, 2016.

[13] A. Ghasemi, R. Kazemi, and S. Azadi, "Stable decentralized control of a platoon of vehicles with heterogeneous information feedback," IEEE Transactions on Vehicular Technology, vol. 62, no. 9, pp. 4299-4308, 2013.

[14] L. Xiao and F. Gao, "Practical string stability of platoon of adaptive cruise control vehicles," IEEE Transactions on Intelligent Transportation Systems, vol. 12, no. 4, pp. 1184-1194, 2011.

[15] S. Sheikholeslam and C. A. Desoer, "Longitudinal control of a platoon of vehicles with no communication of lead vehicle information: a system level study," IEEE Transactions on Vehicular Technology, vol. 42, no. 4, pp. 546-554, 1993.

[16] J. Si and C. Chin, "An adaptable walking-skid for seabed ROV under strong current disturbance," Journal of Marine Science and Application, vol. 13, no. 3, pp. 305-314, 2014.

[17] C. S. Chin, M. W. S. Lau, and E. Low, "Supervisory cascaded controller design: experiment test on a remotely operated vehicle," Proceedings of the Institution of Mechanical Engineers, Part C: Journal of Mechanical Engineering Science, vol. 225, no. 3, pp. 584-603, 2011.

[18] R. Miller and M. Pachter, "Maneuvering flight control with actuator constraints," Journal of Guidance, Control, and Dynamics, vol. 20, no. 4, pp. 729-734, 1997.
[19] S. Gao, H. Dong, Y. Chen, B. Ning, G. Chen, and X. Yang, "Approximation-based robust adaptive automatic train control: an approach for actuator saturation," IEEE Transactions on Intelligent Transportation Systems, vol. 14, no. 4, pp. 1733-1742, 2013.

[20] G. Indiveri, J. Paulus, and P. Plöger, "Motion control of swedish wheeled mobile robots in the presence of actuator saturation," RoboCup 2006: Robot Soccer World Cup X, pp. 35-46, 2007.

[21] B. Xiao, Q. Hu, and Y. Zhang, "Adaptive sliding mode fault tolerant attitude tracking control for flexible spacecraft under actuator saturation," IEEE Transactions on Control Systems Technology, vol. 20, no. 6, pp. 1605-1612, 2012.

[22] B. Zhang, S. Song, Z. Zheng, and X. Wei, "Distributed coordinated formation control of multiple spacecraft with and without velocity measurements," in Proceedings of the 31st Chinese Control Conference (CCC '12), pp. 6105-6111, July 2012.

[23] T. A. Jesus, L. C. A. Pimenta, L. A. B. Tôrres, and E. M. A. M. Mendes, "Consensus for double-integrator dynamics with velocity constraints," International Journal of Control, Automation and Systems, vol. 12, no. 5, pp. 930-938, 2014.

[24] R. Cui, L. Chen, C. Yang, and M. Chen, "Extended state observer-based integral sliding mode control for an underwater robot with unknown disturbances and uncertain nonlinearities," IEEE Transactions on Industrial Electronics, vol. 64, no. 8, pp. 6785-6795, 2017.

[25] A. K. Bondhus, K. Y. Pettersen, and J. T. Gravdahl, "Leader/Follower synchronization of satellite attitude without angular velocity measurements," in Proceedings of the 44th IEEE Conference on Decision and Control, and the European Control Conference (CDC-ECC '05), pp. 7270-7277, December 2005.

[26] C. S. Chin, "Systematic modeling and model-based simulation of a remotely operated vehicle using matlab and simulink," International Journal of Modeling, Simulation, and Scientific Computing, vol. 2, no. 4, pp. 481-511, 2011.

[27] Y. Li, S. Tong, and T. Li, "Direct adaptive fuzzy backstepping control of uncertain nonlinear systems in the presence of input saturation," Neural Computing and Applications, vol. 23, no. 5, pp. 1207-1216, 2013.

[28] A. Abdessameud and A. Tayebi, "Attitude synchronization of a group of spacecraft without velocity measurements," Institute of Electrical and Electronics Engineers. Transactions on Automatic Control, vol. 54, no. 11, pp. 2642-2648, 2009.

[29] A. Abdessameud and A. Tayebi, "Velocity-free consensus algorithms for double-integrator dynamics with input saturations constraints," in Proceedings of the 2010 49th IEEE Conference on Decision and Control (CDC '10), pp. 4486-4491, December 2010. 


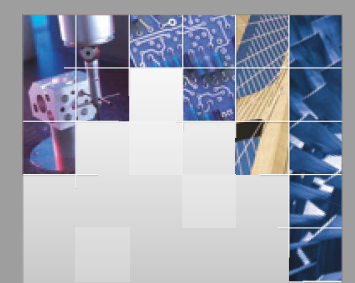

\section{Enfincering}
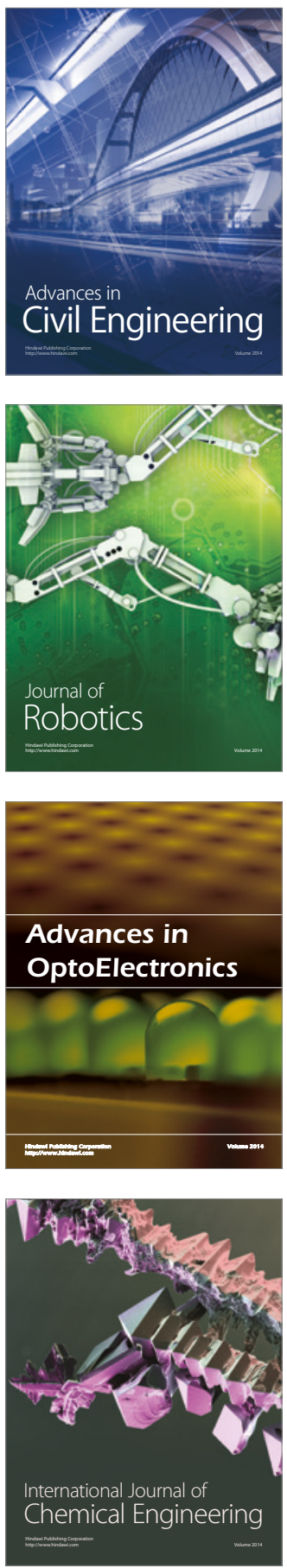

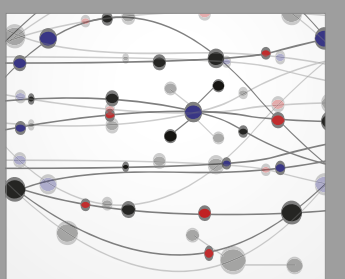

The Scientific World Journal

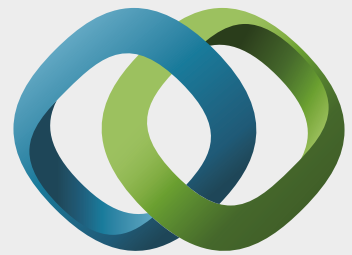

\section{Hindawi}

Submit your manuscripts at

https://www.hindawi.com
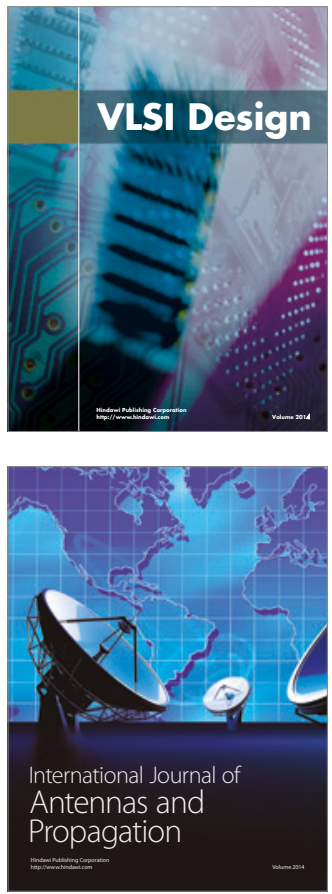

\section{Rotating}

Machinery
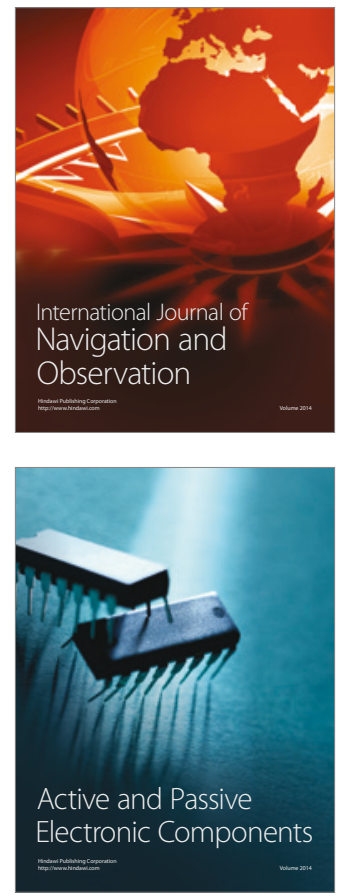
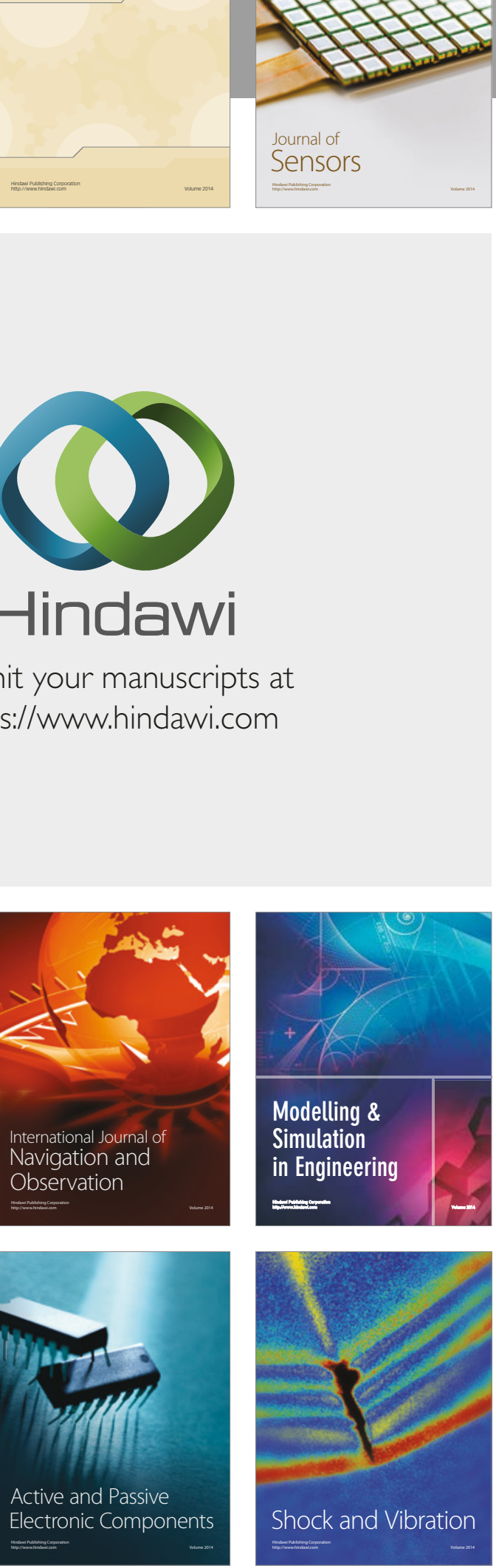
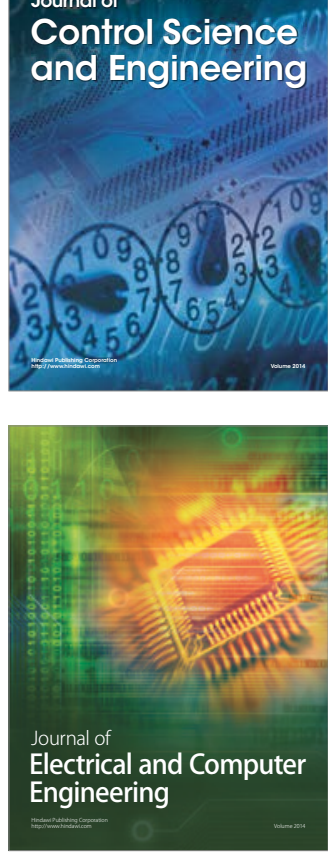

Distributed

Journal of

Control Science

and Engineering
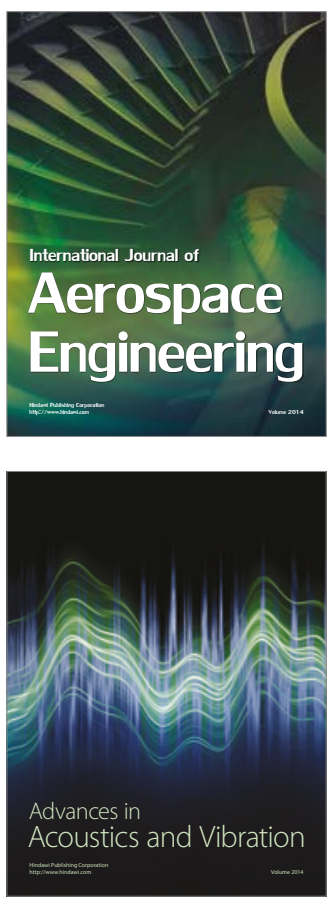

Sensor Networks 